\title{
Change in Energy of Non-Spinning Black Holes w.r.t. the Change in Mass
}

\author{
Dipo Mahto ${ }^{1}$, Rama Nand Mehta ${ }^{2}$, Neeraj Pant ${ }^{3}$, Raj Kumar Sah ${ }^{4}$ \\ ${ }^{1}$ Department of Physics, Marwari College, T. M. B. U., Bhagalpur, India \\ ${ }^{2}$ University Department of Mathematics, T. M. B. U., Bhagalpur, India \\ ${ }^{3}$ Department of Mathematics, N. D. A., Khadakwasala, Pune, India \\ ${ }^{4}$ Department of Physics, S. S. V. College, Kahalgaon, T. M. B. U., Bhagalpur, India \\ Email: dipomahto@hotmail.com
}

Received November 23, 2012; revised December 26, 2012; accepted January 11, 2013

\begin{abstract}
The present work discusses the derivation of the formula for the change in energy of non-spinning black holes with respect to the change in mass $(\mathrm{d} E / \mathrm{d} M)$, which gives a constant quantity equal to $8.9998 \times 10^{16} \mathrm{Joule} / \mathrm{kg}$ in both categories of X-ray binaries (XRBs) and Active Galactic Nuclei (AGN). This formula can be used to justify the life time of black hole given by $\Gamma=2.098\left(M / M_{\odot}\right)^{3} \times 10^{67}$ years as proposed by Stephen Hawking, where $M$ and $M_{\odot}$ are the mass of the black hole and the sun respectively. The authors also calculate the change in energy and mass of non-spinning black holes with respect to the change in the radius of event horizon as well as $(\mathrm{d} E / \mathrm{d} M)$ for different test non-spinning black holes in X-ray binaries (XRBs) and Active Galactic Nuclei (AGN).
\end{abstract}

Keywords: Life Time; XRBs; AGN; Energy

\section{Introduction}

When stars are born, they form from existing gas dust. This is called interstellar matter. When cloud of interstellar matter crosses the spiral arm of a galaxy, it begins to form clumps. The gravitational forces within the clumps cause them to contract, forming protostar. The centre of a protostar may reach a temperature of a several million of degree Celsius. At this high temperature, a fusion reaction begins. The energy released by this reaction prevents the protostar to contract. Thus, a star has been formed. There are so many stages of a star from its birth to death. The black hole is the final stage of dying star having masses $5 M_{\odot}-20 M_{\odot}$ found in X-ray binaries (XRBs) and $10^{6} M_{\odot}-10^{9.5} M_{\odot}$ found in Active Galactic Nuclei (AGN) where $M_{\odot}$ stands for the mass of the sun [1]. Like a living creature, the star takes birth and finally dies. The living creatures are very short-lived than that of the stellar bodies.

Classically, the black holes are perfect absorbers but do not emit anything; their temperature is absolute zero [2]. But in 1974, Stephan Hawking showed that quantum theory predicts that the black hole should radiate energy like a black body with a temperature proportional to the surface gravity of the black hole [3] and sometimes also after the physicists, Jacob Bekenstein who predicted that black holes should have a finite, non-zero temperature and entropy [4].

In 2010, Kanak Kumari et al derived an expression for the energy of non-spinning black holes in terms of the radius of event horizon $\left(E_{B H}=K_{B H} R_{s}\right)$ where $K_{B H}$ is the Black hole constant for non-spinning black holes having value equal to $6.0711 \times 10^{43} \mathrm{~J} \cdot \mathrm{m}^{-1}$ [5].

In 2011, Dipo Mahto et al derived an expression for the energy of spinning black holes in terms of the radius of event horizon $\left(E_{B H s}=K_{B H s} R_{s}^{\prime}\right)$ where $K_{B H s}$ is the black hole constant for spinning black holes having value equal to $1.214 \times 10^{44} \mathrm{~J} \cdot \mathrm{m}^{-1}$ [6]. In the same year Dipo Mahto et al derived the formula for both the changes in energy $(\delta E)$ and entropy $(\delta S)$ and calculated their values for different test non-spinning black holes in XRBs and AGN [7].

In the present research work, we have derived the formula for the change in energy of non-spinning black holes with respect to the change in mass $(\mathrm{d} E / \mathrm{d} M)$, which gives a constant quantity equal to $8.9998 \times 10^{16}$ Joule $/ \mathrm{kg}$ in both categories of X-ray binaries (XRBs) and Active Galactic Nuclei (AGN), where $M$ and $M_{\odot}$ are the mass of the black hole and the sun respectively. This formula can be used to justify the life time of black hole given by $\Gamma=2.098\left(M / M_{\odot}\right)^{3} \times 10^{67}$ years as proposed 
by Stephen Hawking. We have also calculated the change in energy and mass of non-spinning black holes with respect to the change in the radius of event horizon as well as $(\mathrm{d} E / \mathrm{d} M)$ for different test non-spinning black holes in X-ray binaries (XRBs) and Active Galactic Nuclei (AGN).

\section{Discussion}

\subsection{Black Hole}

A black hole is a solution of Einstein's gravitational field equations in the absence of matter that describes the space time around a gravitationally collapsed star. Its gravitational pull is so strong that even light can not escape from it [8].

Hawking radiation is a thermal radiation with a black body spectrum predicted to be emitted by black holes due to quantum effects. The Hawking radiation process reduces the mass and energy of the black hole and therefore also known as black hole evaporation. The black holes that lose more matter than they gain through other means are expected to dissipate, shrink, and ultimately vanish. Smaller micro black holes are predicted to be larger net emitters of radiation than larger black holes, they tend to shrink and dissipate faster [3].

\subsection{Expression for $d E / d M$}

To discuss the loss in mass and energy of black holes, Einstein mass-energy equivalence relation can be applied with the first law of black hole mechanics. The Einstein mass-energy equivalence relation is given by [9].

$$
E=M c^{2}
$$

The change in energy of black holes taking place the change in their mass as

$$
\mathrm{d} E=\mathrm{d} M \cdot c^{2}
$$

The change in energy of the black holes $(\mathrm{d} E)$ can be calculated when the change in mass of the black holes $(\mathrm{d} M)$ are known. For this the first law of black hole is applied such as.

The first law of black hole mechanics is mass, $M$, angular momentum, $J$, and horizon area, $A$, of a stationary black hole when it is perturbed. To first order, the variations of these quantities in the vacuum case always satisfy [2].

$$
\mathrm{d} M=\frac{1}{8 \pi} \kappa \mathrm{d} A+\Omega \mathrm{d} J
$$

In the case of non-spinning black holes, $J=0$, and hence $\mathrm{d} J=0[1,7]$.

The Equation (3) takes its form as

$$
\mathrm{d} M=\frac{1}{8 \pi} \kappa \mathrm{d} A
$$

For the spherically, symmetric and stationary, or Schwarzschild black hole's mass $M$, the horizon's radius is $R_{b h}=2 G M / c^{2}$ and its area is naturally given by the following relation $[7,10,12]$.

$$
A=4 \pi R_{b h}^{2}
$$

where $R_{b h}$ stands for Schwarzschild radius of horizon area of a black hole. Differentiating Equation (5), we have

$$
\mathrm{d} A=8 \pi R_{b h} \mathrm{~d} R_{b h}
$$

Putting the above value in Equation (4) and solving, we have

$$
\mathrm{d} M=\kappa R_{b h} \mathrm{~d} R_{b h}
$$

or

$$
\mathrm{d} M / \mathrm{d} R_{b h}=\kappa R_{b h}
$$

In Equation (8), the term $\kappa$ is the surface gravity of black holes. For the Schwarzschild case, the surface gravity $(\kappa)$ of a black hole is given by $[11,12]$.

$$
\kappa=\frac{1}{4 M}
$$

The term $M$ stands for the mass of black holes.

From Equation (9), it is clear that the surface gravity of black hole is inversely proportional to its mass and the different black holes will have different surface gravity. Greater the mass of the black holes, smaller the surface gravity and vice-versa.

The surface gravity $(\kappa)$ has the same role in the black hole mechanics as the temperature in the ordinary laws of thermodynamics. The zeroth law of classical black hole mechanics states that the surface gravity $(\kappa)$ of a black hole is constant on horizon. The surface gravity tends to zero when the magnitude of charge of a black holes becomes equal to the mass of black holes $[11,12]$ and the surface gravity $(\kappa)$ can be thought of roughly as the acceleration at horizon of black hole [8]. Putting the value of $\mathrm{d} M$ in Equation (2), we have

$$
\mathrm{d} E=\kappa R_{b h} \mathrm{~d} R_{b h} c^{2}
$$

Or

$$
\mathrm{d} E / \mathrm{d} R_{b h}=\kappa R_{b h} c^{2}
$$

Equation (11) can be used to calculate the change in energy of black holes with respect to the radius of event horizon.

Using the Equation (9), the Equations (8) and (11) can be written as

$$
\begin{aligned}
& \mathrm{d} M / \mathrm{d} R_{b h}=R_{b h} / 4 M \\
& \mathrm{~d} E / \mathrm{d} R_{b h}=R_{b h} c^{2} / 4 M
\end{aligned}
$$

The Equations (12) and (13) can be used to determine 
the change in mass and energy with respect to change in radius of event horizon of different test non-spinning black holes respectively. Now the Equation (13) is divided by Equation (12), we get

$$
\frac{\mathrm{d} E}{\mathrm{~d} M}=c^{2}
$$

The Equation (14) shows the change in energy of different test non-spinning black holes with respect to the change in mass and has vital role to justify life time of black holes $\Gamma=2.098\left(M / M_{\odot}\right)^{3} \times 10^{67}$ years given by Stephan Hawking.

\subsection{Data in Support of Mass of BHs}

There are two categories of Black holes classified on the basis of their masses clearly very distinct from each other, with very different masses $M \sim 5-20 M_{\odot}$ for stellar - mass Black holes in X-ray binaries and $M \sim 10^{6}-10^{9.5}$
$M_{\odot}$ in Active Galactic Nuclei [1,11].

Here we have calculated the change in mass and energy with respect to the radius of event horizon using Equations (10) and (11) respectively as well as $(\mathrm{d} E / \mathrm{d} M)$ for different test Non-spinning black holes in X-ray binaries (XRBs) and Active Galactic Nuclei (AGN) listed in Table 1.

\section{Result and Discussion}

In the present work, we have derived the formula for the change in energy of non-spinning black holes with respect to the change in mass $(\mathrm{d} E / \mathrm{d} M)$ giving a constant quantity, which can be used to justify the life time of black hole in both categories X-ray binaries (XRBs) and Active Galactic Nuclei (AGN). We have calculated the change in mass $(d M)$ and energy $(d E)$ with respect to change in the radius of event horizon as well as $(\mathrm{d} E / \mathrm{d} M)$ for different test non-spinning black holes in

Table 1. $M_{\odot}=2 \times 10^{30} \mathrm{~kg}$. Change in energy with respect to change in mass of non-spinning black holes.

\begin{tabular}{|c|c|c|c|c|c|c|}
\hline Sl. No. & $\begin{array}{l}\text { Mass of } \\
\text { BHs }(M)\end{array}$ & $\begin{array}{c}\text { Radius of event horizon } \\
\text { of BHs } \quad\left(R_{b h}=2950 M / M_{\odot}\right) \\
(\text { in metre })\end{array}$ & $\begin{array}{l}\text { Change in mass of } \\
\text { non-spinning black holes } \\
\left.\mathrm{d} M=\frac{R_{b h}}{4 M} \quad \text { (in } \mathrm{kg}\right)\end{array}$ & $\begin{array}{l}\text { Change in energy of } \\
\text { non-spinning black holes } \\
\mathrm{d} E=\frac{R_{b h}}{4 M} \cdot c^{2} \quad \text { (in Joule) }\end{array}$ & $\begin{array}{l}\text { Change in energy } \\
\text { w.r.t. change in mass } \\
\frac{\mathrm{d} E}{\mathrm{~d} M}=c^{2} \quad \text { (in } \mathrm{J} / \mathrm{kg} \text { ) }\end{array}$ & \\
\hline 1 & $5 M_{\odot}$ & 14750 & $3.6875 \times 10^{-28}$ & $3.3187 \times 10^{-11}$ & $8.9998 \times 10^{16}$ & \multirow{21}{*}{ XRBs } \\
\hline 2 & $6 M_{\odot}$ & 17700 & $3.6875 \times 10^{-28}$ & $3.3187 \times 10^{-11}$ & $8.9998 \times 10^{16}$ & \\
\hline 3 & $7 M_{\odot}$ & 20650 & $3.6875 \times 10^{-28}$ & $3.3187 \times 10^{-11}$ & $8.9998 \times 10^{16}$ & \\
\hline 4 & $8 M_{\odot}$ & 23600 & $3.6875 \times 10^{-28}$ & $3.3187 \times 10^{-11}$ & $8.9998 \times 10^{16}$ & \\
\hline 5 & $9 M_{\odot}$ & 26550 & $3.6875 \times 10^{-28}$ & $3.3187 \times 10^{-11}$ & $8.9998 \times 10^{16}$ & \\
\hline 6 & $10 M_{\odot}$ & 29500 & $3.6875 \times 10^{-28}$ & $3.3187 \times 10^{-11}$ & $8.9998 \times 10^{16}$ & \\
\hline 7 & $11 M_{\odot}$ & 32450 & $3.6875 \times 10^{-28}$ & $3.3187 \times 10^{-11}$ & $8.9998 \times 10^{16}$ & \\
\hline 8 & $12 M_{\odot}$ & 35400 & $3.6875 \times 10^{-28}$ & $3.3187 \times 10^{-11}$ & $8.9998 \times 10^{16}$ & \\
\hline 9 & $13 M_{\odot}$ & 38350 & $3.6875 \times 10^{-28}$ & $3.3187 \times 10^{-11}$ & $8.9998 \times 10^{16}$ & \\
\hline 10 & $14 M_{\odot}$ & 41300 & $3.6875 \times 10^{-28}$ & $3.3187 \times 10^{-11}$ & $8.9998 \times 10^{16}$ & \\
\hline 11 & $15 M_{\odot}$ & 44250 & $3.6875 \times 10^{-28}$ & $3.3187 \times 10^{-11}$ & $8.9998 \times 10^{16}$ & \\
\hline 12 & $16 M_{\odot}$ & 47200 & $3.6875 \times 10^{-28}$ & $3.3187 \times 10^{-11}$ & $8.9998 \times 10^{16}$ & \\
\hline 13 & $17 M_{\odot}$ & 50150 & $3.6875 \times 10^{-28}$ & $3.3187 \times 10^{-11}$ & $8.9998 \times 10^{16}$ & \\
\hline 14 & $18 M_{\odot}$ & 53100 & $3.6875 \times 10^{-28}$ & $3.3187 \times 10^{-11}$ & $8.9998 \times 10^{16}$ & \\
\hline 15 & $19 M_{\odot}$ & 56050 & $3.6875 \times 10^{-28}$ & $3.3187 \times 10^{-11}$ & $8.9998 \times 10^{16}$ & \\
\hline 16 & $20 M_{\odot}$ & 59000 & $3.6875 \times 10^{-28}$ & $3.3187 \times 10^{-11}$ & $8.9998 \times 10^{16}$ & \\
\hline 17 & $10^{6} M_{\odot}$ & $2.95 \times 10^{9}$ & $3.6875 \times 10^{-28}$ & $3.3187 \times 10^{-11}$ & $8.9998 \times 10^{16}$ & \\
\hline 18 & $10^{7} M_{\odot}$ & $2.95 \times 10^{10}$ & $3.6875 \times 10^{-28}$ & $3.3187 \times 10^{-11}$ & $8.9998 \times 10^{16}$ & \\
\hline 19 & $10^{8} M_{\odot}$ & $2.95 \times 10^{11}$ & $3.6875 \times 10^{-28}$ & $3.3187 \times 10^{-11}$ & $8.9998 \times 10^{16}$ & \\
\hline 20 & $10^{9} M_{\odot}$ & $2.95 \times 10^{12}$ & $3.6875 \times 10^{-28}$ & $3.3187 \times 10^{-11}$ & $8.9998 \times 10^{16}$ & \\
\hline 21 & $10^{9.5} M_{\odot}$ & $2.95 \times 10^{12.5}$ & $3.6875 \times 10^{-28}$ & $3.3187 \times 10^{-11}$ & $8.9998 \times 10^{16}$ & \\
\hline
\end{tabular}


X-ray binaries (XRBs) and Active Galactic Nuclei (AGN) and the graphs have been plotted between:

1) the radius of event horizon $\left(R_{b h}\right)$ of different test black holes and their corresponding values of change in mass in XRBs (Figure 1);

2 ) the radius of event horizon $\left(R_{b h}\right)$ of different test black holes and their corresponding values of change in mass in AGN (Figure 2);

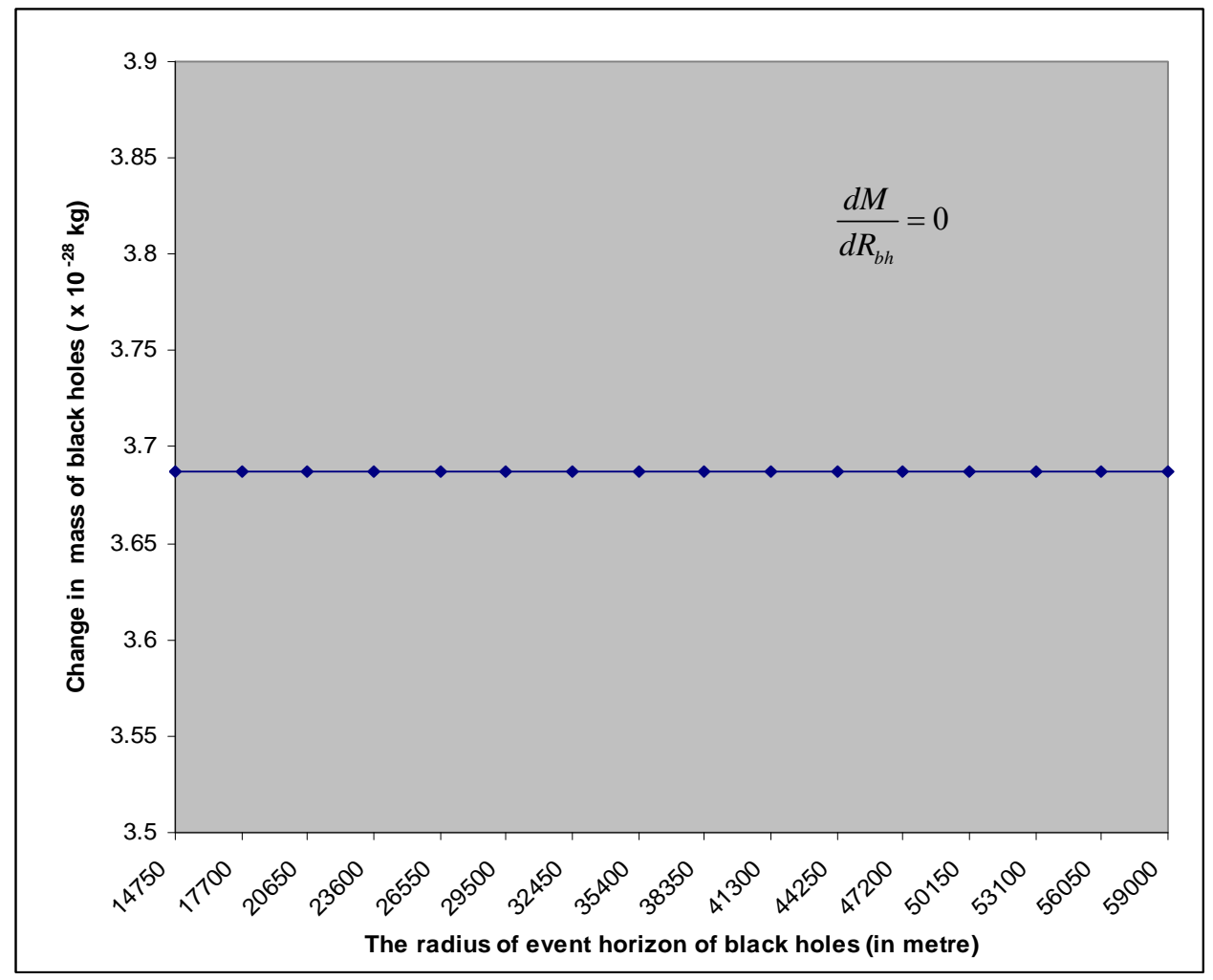

Figure 1. The graph plotted between the radius of event horizon $\left(R_{b h}\right)$ of different BHs and their corresponding values of change in mass in XRBs.

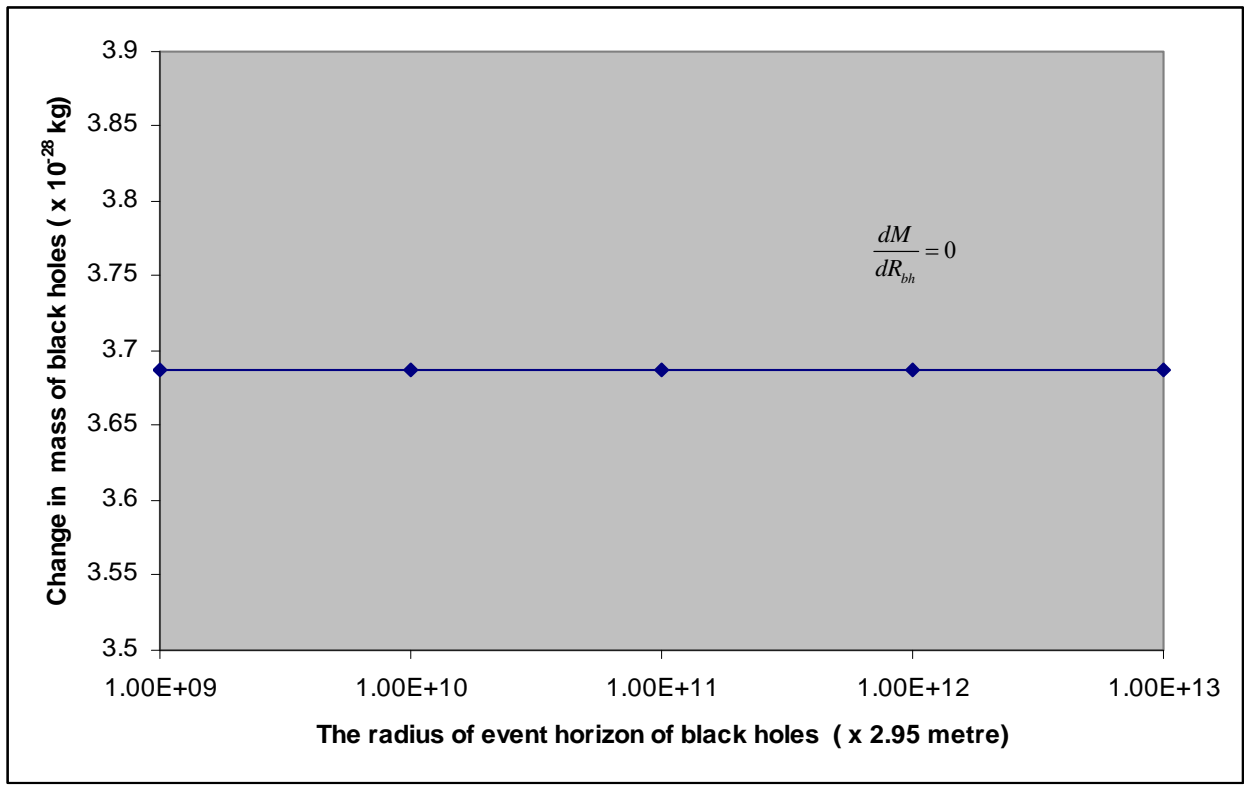

Figure 2. The graph plotted between the radius of event horizon $\left(R_{b h}\right)$ of different BHs and their corresponding values of change in mass in AGN. 
3) the radius of event horizon of different test black holes and their corresponding values in the change in energy in XRBs (Figure 3);

4) the radius of event horizon of different test black holes and their corresponding values in the change in energy in AGN (Figure 4);

5) the radius of event horizon of different test black holes and their corresponding values of $(\mathrm{d} E / \mathrm{d} M)$ in

\section{XRBs (Figure 5);}

6) the radius of event horizon of different test black holes and their corresponding values of $(\mathrm{d} E / \mathrm{d} M)$ in AGN (Figure 6).

The Figures 1 and 2 shows that the change in mass of non-spinning black holes remain the same with the increase the radius of event horizon of the different test non-spinning black holes in both XRBs and AGN

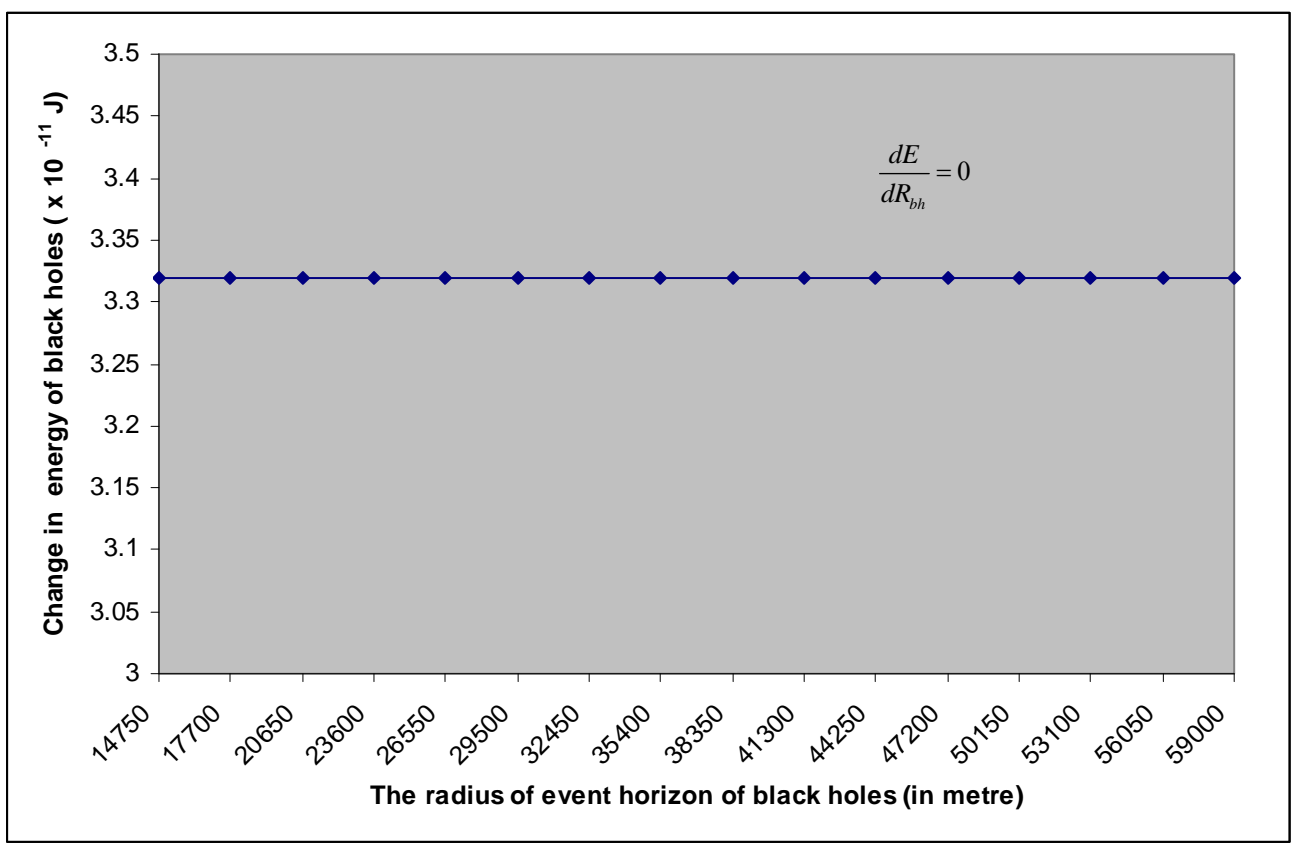

Figure 3. The graph plotted between the radius of event horizon $\left(R_{b h}\right)$ of different BHs and their corresponding values of change in mass in XRBs.

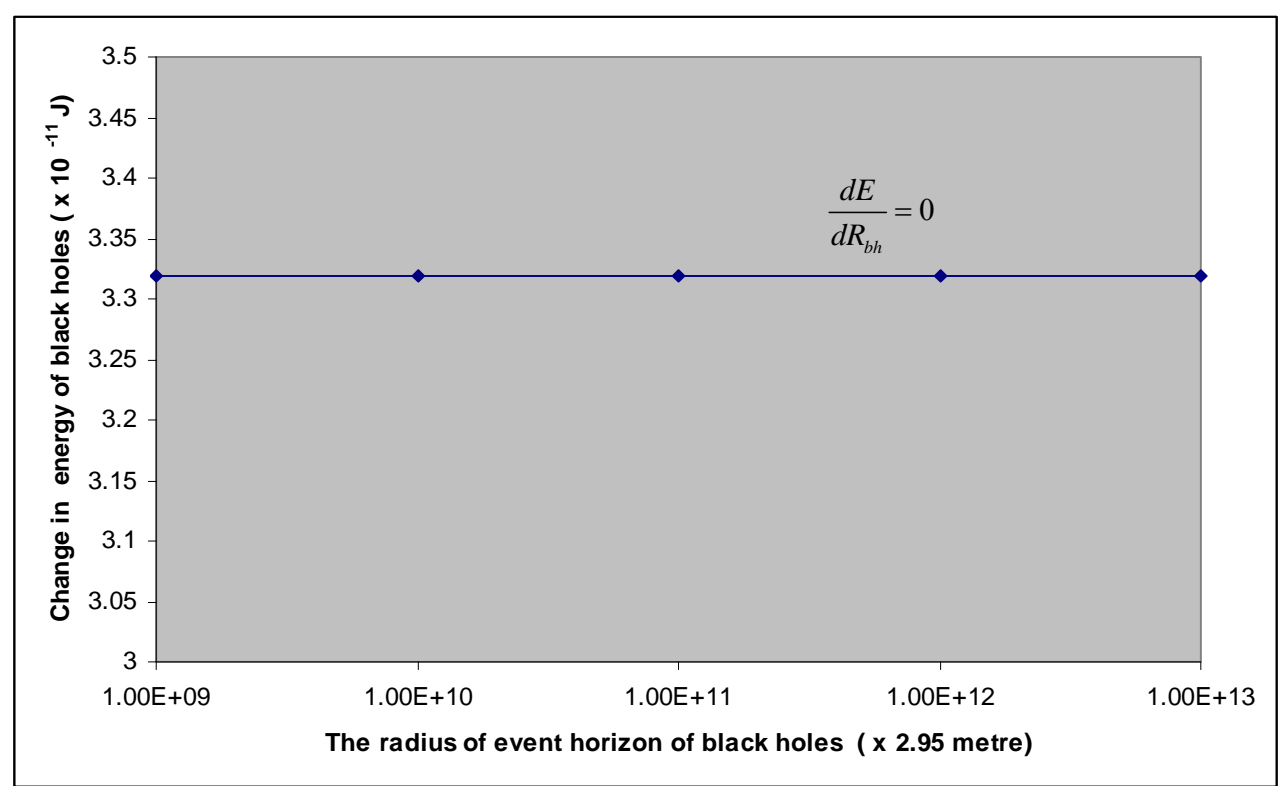

Figure 4. The graph plotted between the radius of event horizon $\left(\boldsymbol{R}_{b h}\right)$ of different BHs and their corresponding values of change in mass in AGN. 


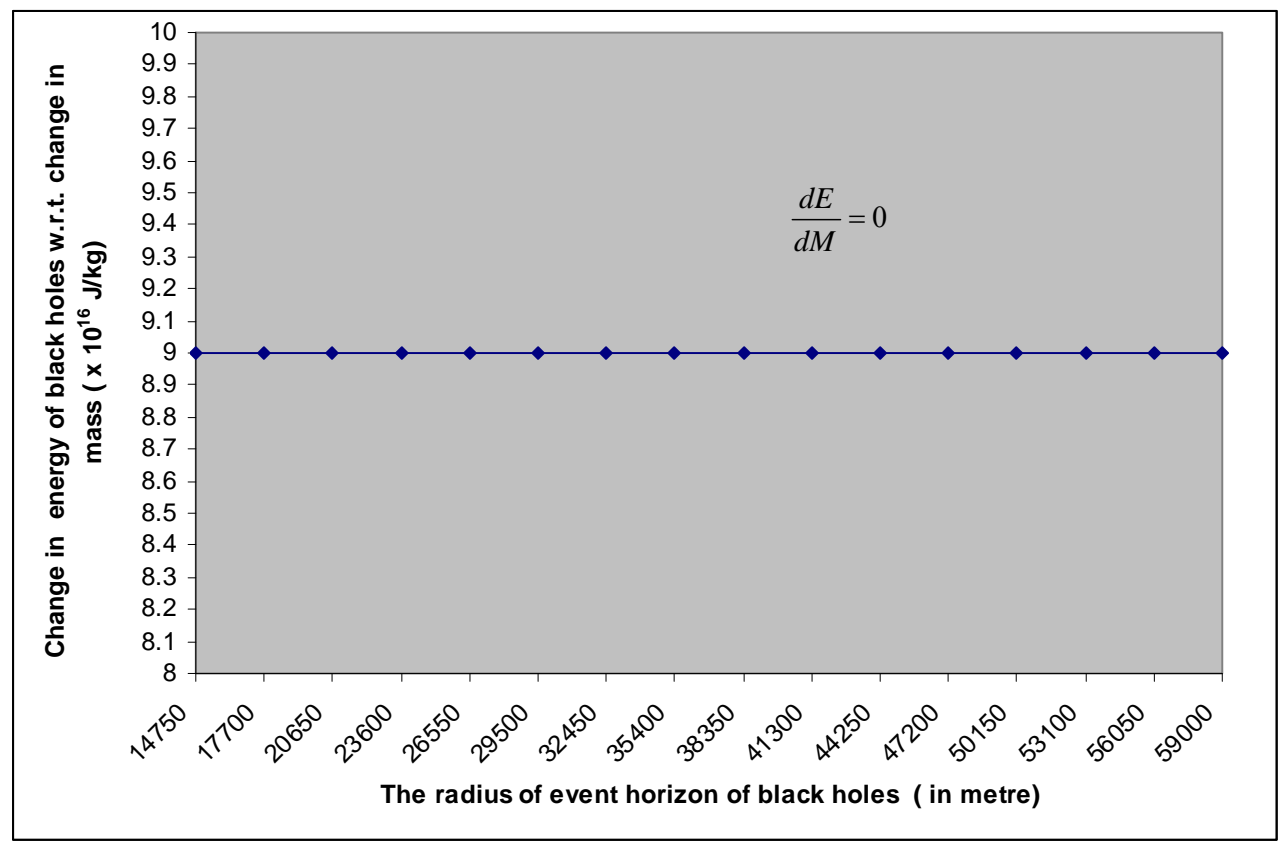

Figure 5. The graph plotted between the radius of event horizon and change in energy of black holes w.r.t. change in mass in XRBs.

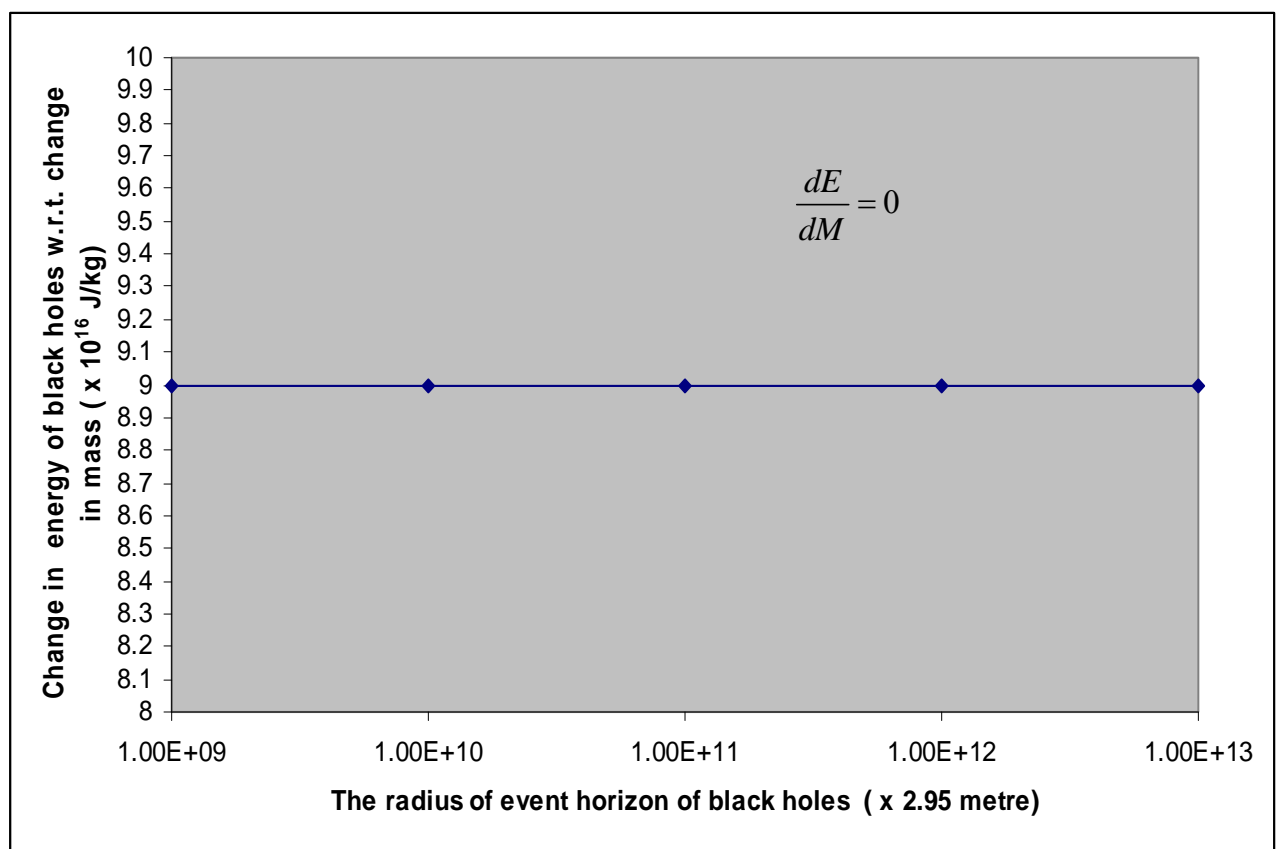

Figure 6. The graph plotted between the radius of event horizon and change in energy of black holes w.r.t. change in mass in AGN.

respectively. The slope of the graph in Figures $\mathbf{1}$ and $\mathbf{2}$ shows that $\frac{\mathrm{d} M}{\mathrm{~d} R_{b h}}=0$ or $\mathrm{d} M=0$ or $\int \mathrm{d} M=\int 0$ or $M=$ constant .

Hence the systems like black holes obey the principle of conservation of mass.

The calculated data for the change in the mass of non-spinning black holes in each case existing either in $\mathrm{XRBs}$ or AGN is the same, showing the principle of conservation of mass, which is experimental fact.

The Figures $\mathbf{3}$ and $\mathbf{4}$ show that the change in energy of non-spinning black holes remain the same with the increase the radius of event horizon of the different test non-spinning black holes in both XRBs and AGN re- 
spectively. The slope of the graph in Figures 3 and $\mathbf{4}$ show that $\frac{\mathrm{d} E}{\mathrm{~d} R_{b h}}=0$ or $E=$ constant .

Hence the systems like black holes obey the principle of conservation of energy.

Similarly the calculated data for the change in the energy of non-spinning black holes in each case existing either in XRBs or AGN is the same showing the principle of conservation of energy, which is also experimental fact.

The Figures 5 and $\mathbf{6}$ show that the change in energy with respect to the change in mass of non-spinning black holes remains the same with the increase the radius of event horizon of the different test non-spinning black holes in both XRBs and AGN respectively. The slope of the graph in Figures 5 and 6 show that $\frac{\mathrm{d} E}{\mathrm{~d} M}=0$ or $\mathrm{d} E=0$ or $\int \mathrm{d} E=\int 0$ or $E=$ constant.

Hence the systems like black holes obey the principle of conservation of energy. Similarly the calculated data for the change in the energy of non-spinning black holes in each case existing either in XRBs or AGN is the same showing the principle of conservation of energy, which is also experimental fact.

\section{Justification of Life Time of BHs}

The formula for life time of the black holes as proposed by Stephen Hawking is given by $\Gamma=2.098\left(M / M_{\odot}\right)^{3} \times 10^{67}$ years. The equation for life time of black holes shows that the life time will be longer for heavier black holes than that of lighter black holes. In the present research work, we have shown that the rate of emission of energy with respect to mass $(\mathrm{d} E / \mathrm{d} M)$ is constant for all black holes either existing in XRBs or AGN. This means that heavier and lighter black holes radiate energy in the same amount. The loss in energy of non-spinning black holes due to radiation of energy will reduce the mass of black holes according to Einstein mass-energy equivalence relation $\left(E=m c^{2}\right)$. This results that the heavier black holes will take longer time to reduce their masses completely into energy radiated than that of the lighter black holes. Hence the heavier black holes will have longer life time than that of lighter. This result is good agreement with the formula for life time of black holes $\Gamma=2.098\left(M / M_{\odot}\right)^{3} \times 10^{67}$ years given by Stephan Hawking. This justifies the life time of black holes.

\section{Conclusions}

In the present study of present research work, we can draw the following conclusion:

1) The change in mass calculated for each case non-spinning black hole gives the constant values equal to $3.6875 \times 10^{-28} \mathrm{~kg}$ in the both categories of black holes existing in XRBs and AGN leading the principle of conservation of mass;

2) The change in energy calculated for each case non-spinning black hole gives the constant values equal to $3.3187 \times 10^{-11}$ joule in the both categories of black holes existing in XRBs and AGN leading the principle of conservation of energy;

3) The change in energy with respect to change in mass $(\mathrm{d} E / \mathrm{d} M)$ calculated for each case non-spinning black hole gives the constant values equal to $8.9998 \times 10^{16}$ joule per $\mathrm{kg}$ in the both categories of black holes either existing in X-ray binary (XRBs) or Active Galactic Nuclie (AGN) justifying the life time of black holes that the heavier black holes will have longer life time than that of lighter. This result is good agreement with the formula $\Gamma=2.098\left(M / M_{\odot}\right)^{3} \times 10^{67}$ years.

\section{Acknowledgements}

Authors acknowledge their gratitude to Dr. Gopi Kant Jha, Former Head, Department of Physics, L. N. M. U. Darbhanga (Bihar), Dr. Kamal Prasad, Dr. B. N. Singh, Dr. Ashutosh Prasad, Associate Professor, University Department of Physics, T. M. B. U. Bhagalpur and Dr. M. S. H. "John", Principal Marwari College Bhagalpur for their stimulating discussion and motivation. Authors are also grateful to the referee for pointing out the errors in the original manuscript and making constructive suggestions.

\section{REFERENCES}

[1] R. Narayan, "Black Holes in Astrophysics," New Journal of Physics, Vol. 7, No. 1, 2005, pp. 1-31.

[2] R. M. Wald, "The Thermodynamics of Black Holes. Living Reviews in Relativity,” 2001.

[3] S. W. Hawking, “A Black Hole Explosion?” Nature, Vol. 248, 1974, pp. 30-31.

http://www.nature.com/nature/journal/v248/n5443/abs/24 8030a0.html

[4] C. Rose, "A Conversation with Dr. Stephen Hawking and Lucy Hawking". http://www.charlierose.com/guest/view/6294

[5] K. Kumari, D. Mahto, G. Chandra, R. K. Sah and K. M. Singh, "Study of Schwarzschild's Radius with Reference to the Non-Spinning Black Holes," Bulletin of Pure and Applied Sciences, Vol. 29D, No. 2, 2010, pp. 183-187.

[6] D. Mahto, K. Kumari, R. K. Sah and V. Prakash, "Study of Schwarzschild's Radius with Reference to the Spinning Black Holes," Bulletin of Pure and Applied Sciences, Vol. 30D, No. 1, 2011, pp. 157-162.

[7] D. Mahto, K. Kumari, R. K. Sah and K. M. Singh, "Study of Non-Spinning Black Holes with Reference to the Change in Energy and Entropy," Astrophysics and Space 
Science, Vol. 337, No. 2, 2011, pp. 685-691. doi:10.1007/s10509-011-0883-7

[8] A. Dabholkar, "Black Hole Entropy in String Theory. A Window in to the Quantum Structure of Gravity," Current Science, Vol. 89, No. 12, 2005, p. 25.

[9] P. G. Bergmann, "Introduction to the Theory of Relativity," Prentice-Hall of India, New Delhi, 1969.

[10] J. D. Bekenstein, "Bekenstein-Hawking Entropy," Scho- larpedia, Vol. 3, No. 10, 2008, p. 7375

[11] D. Mahto, V. Prakash, B. K. Singh and K. M. Singh, "Change in Entropy of Non-Spinning Black Holes w.r.t. the Radius of Event Horizon in XRBs," Astrophysics and Space Science, Vol. 343, No. 1, 2013, pp. 153-159. doi:10.1007/s10509-012-1219-y

[12] J. Transchen, "An Introduction to Black Hole Evaporation," 2000. 